\title{
Documentos
}

\section{Recomendaciones en la prevencion secundaria de Cardiopatía isquémica en la mujer}

\author{
Paola Varleta ${ }^{1,2}$, Monica Acevedo ${ }^{2,3}$, Giovanna Valentino $3,4, a, b$, Rosario López 1,2,c,d \\ 1. Unidad de Prevención Cardiovascular y Rehabilitación Cardiaca, Centro Cardiovascular, Hospital DIPRECA, Santiago, Chile. \\ 2. Departamento de Prevención y Rehabilitación Cardiovascular, Sociedad Chilena de Cardiología y Cirugía Cardiovascular. \\ 3. División de Enfermedades Cardiovasculares, Facultad de Medicina, Pontificia Universidad Católica de Chile, Santiago, Chile. \\ 4. Carrera de Nutrición y Dietética, Departamento de Ciencias de la Salud, Facultad de Medicina, Pontificia Universidad Católica de Chile, \\ Santiago, Chile. \\ a. Magister Fisiología Humana. \\ b. Nutricionista. \\ c. Magister Fisiología del Ejercicio. \\ d. Kinesióloga.
}

La enfermedad cardiovascular en la mujer es la principal causa de muerte en Chile. La mujer con cardiopatía isquémica coronaria representa un riesgo de mortalidad dos veces mayor que el del hombre. Desgraciadamente, las mujeres han sido subrepresentadas en la mayoría de los estudios clínicos randomizados en prevención secundaria, y más aún, son pocos los que presentan análisis específicos de género en cuanto a la terapia y los puntos finales duros. Así, la evidencia que existe ha sido dirigida especialmente a hombres. Para reducir esta brecha, presentamos una revisión de la información en las distintas terapias en prevención secundaria de cardiopatía isquémica, destacando los resultados de trabajos en que se hizo análisis género-específico.

Palabras Clave: Enfermedad Cardiovascular, Prevención Secundaria, Mujeres, Factores de Riesgo.

\section{Secondary prevention of ischemic heart disease in women}

Cardiovascular disease is the main cause of death in Chilean women. Ischemic heart disease mortality rate in women is two times that of in men. Unfortunately, there have been less enrollment of women in clinical cardiovascular trials and a lack of gender-specific analysis of clinical trial data. Therefore, the evidence for secondary preventive recommendations have been focused on men. To bridge this gap, in this review we address the data for secondary preventive therapies for ischemic heart disease in women assessing the available gender-specific data.

Keywords: Cardiovascular Disease, Secondary Prevention, Women, Risk Factors.

Correspondencia:

Dra. Paola Varleta

pvarleta@manquehue.net.

Dra. Mónica Acevedo

macevedo@med.puc.cl. 


\section{Introducción:}

La enfermedad cardiovascular (CV) es la principal causa de muerte en mujeres en Chile y en países del mundo desarrollado. En Chile, en el 2016 alrededor de un $29 \%$ de las muertes en mujeres fueron atribuidas a la enfermedad $\mathrm{CV}$, un porcentaje incluso, mayor que en hombres ${ }^{1}$. Dentro de la enfermedad CV, la enfermedad isquémica del corazón y la enfermedad cerebrovascular son las principales entidades asociadas a morbimortalidad en la mujer.

Esta revisión se focalizará en la cardiopatía isquémica (CI), término amplio que comprende a condiciones clínicas, tales como la angina crónica, la isquemia silente, y el sindrome coronario agudo (SCA). A la vez, la CI puede tener un sustrato de una enfermedad coronaria obstructiva como no obstructiva. Es importante recordar esto último, porque la mujer tiene vasos coronarios más pequeños en comparación con el hombre, y porque existe una mayor presencia de enfermedad isquémica no obstructiva, secundaria a enfermedad microvascular, la cual es muy sintomática y tiene mal pronóstico $^{2,3}$.

La prevención $\mathrm{CV}$ de cardiopatía coronaria en la mujer es todo un desafío, a la luz de las estadísticas que indican un mayor riesgo $\mathrm{CV}$ de la mujer por sobre el hombre. Es así como en Estados Unidos mueren más mujeres en comparación con hombres dentro del primer año post infarto al miocardio, independiente de la edad $^{4}$. Por otra parte, en Canadá, se ha reportado en sujetos con revascularización percutánea post SCA, una frecuencia de muerte o infarto recurrente al año de 10.6 $\%$ en hombres versus $13.1 \%$ en mujeres ${ }^{5}$. Este incremento en el riesgo no sólo se debe a los factores intrínsecos femeninos, sino lamentablemente también a una menor indicación de tratamiento farmacológico basado en evidencia al alta hospitalaria 6,7 .

La prevención CV es una poderosa arma que incorpora la educación al paciente y la modificación de estilo de vida junto con una terapia farmacológica eficaz. El objetivo primordial de la prevención secundaria es reducir la probabilidad de que aquella mujer que tiene diagnóstico de enfermedad coronaria por imágenes presente un evento coronario, como también prevenir un nuevo evento si ya cursó con uno (SCA y/o revascularización coronaria). De este modo, el seguir las recomendaciones en prevención secundaria y realizar un manejo agresivo de los factores de riesgo $\mathrm{CV}$ es relevante en la mujer. Como medida esencial es el cumplir las recomendaciones de estilo de vida saludable.
Prevención Secundaria en Cardíaca Isquémica: Recomendaciones de Estilo de Vida Saludable.

\section{A. Manejo nutricional y dietario en prevención secun- daria:}

Cabe destacar que en lo referente a la dieta no hay diferencia en las recomendaciones entre ambos géneros. La evidencia es limitada respecto a diversos patrones dietarios y eventos $\mathrm{CV}$. La dieta que mayor evidencia tiene en prevención secundaria es la mediterránea con dos estudios aleatorizados. Se caracteriza por un elevado consumo de alimentos con relación alta de grasas monosaturadas /saturadas (uso de aceite de oliva como ingrediente principal y nueces), alta ingesta de frutas, vegetales y legumbres, adicionalmente de alta ingesta de pescado, granos, frutos secos y acompañado de moderado a bajo consumo de productos lácteos y de alcohol (preferentemente vino tinto en comidas $)^{8,9}$. El principal estudio randomizado es el Lyon Heart Study que comparó la dieta mediterránea con suplemento de canola versus una dieta usual recomendada (Step1 AHA) en 605 pacientes coronarios, mayoritariamente hombres, observando una reducción significativa de mortalidad CV de un 65\% (HR 0.35, 95\% CI 0.15$0.82)^{8}$. Algunos estudios sugieren que los beneficios de la dieta mediterránea se deben a un impacto importante en los parámetros inflamatorios y de función endotelial; no obstante, estudios en pacientes coronarios no han confirmado esos resultados 10,11 . El otro estudio randomizado se ha catalogado de bajo nivel de evidencia, por incorporar un número bajo de sujetos y prescindir de estimación de riesgo ajustado. Por lo anterior, una reciente revisión sistemática del tema, publicada en Cochrane Library, reporta que la dieta mediterránea presenta escasa evidencia en prevención secundaria (Clase IIa, nivel B) ${ }^{12}$.

Es importante ajustar la dieta para que el paciente logre las metas establecidas en factores de riesgo (FRCV): peso aceptable, colesterol LDL $<70 \mathrm{mg} / \mathrm{dL}$ y presión arterial $<140 / 90$, y si se puede $<130 / 80 \mathrm{mmHg} 9$. La dieta mediterránea por sí sola no tiene un alto impacto en peso, LDL o la presión. Se recomienda mantener el peso si es normopeso o bajar de peso (5 a 10\%) lo cual reduce significativamente la presión arterial y la glicemia si el paciente tiene sobrepeso (Clase I, nivel A). Este objetivo se puede lograr con una restricción de 500 kilocalorías diarias dependiendo del peso inicial, y los resultados son independiente del tipo de dieta ${ }^{13,14}$.

Finalmente, la dieta DASH (Dietary Approaches to Stop Hypertension), conocida en el manejo de la hipertensión arterial, no tiene evidencia en prevención secundaria co- 
ronaria. Es una dieta similar a la dieta mediterránea, pero baja en contenido de sodio diario $(100 \mathrm{mEq}=2.3 \mathrm{gr}) \mathrm{y}$ que aporta al menos $3000 \mathrm{mg}$ de potasio diario, logrando reducir la presión sistólica en hasta $11.5 \mathrm{mmHg}$ en hipertensos y $7.1 \mathrm{mmHg}$ en sujetos normotensos. De este modo está indicada en el sujeto coronario hipertenso ${ }^{9}$.

\section{B. Rehabilitación Cardíaca y Ejercicio}

La rehabilitación cardíaca ( $\mathrm{RC}$ ) es un programa integral que incorpora entrenamiento físico, educación sobre control de los FRCV, y motivación al cambio. La RC en lo referente al programa de ejercicio posterior a un SCA y/o una revascularización coronaria, ha demostrado reducir hospitalizaciones y mejorar la calidad de vida de hombres y mujeres, por lo cual tiene en prevención secundaria un nivel de recomendación alta (Clase I, nivel A) ${ }^{9}$. A su vez, Anderson y col ${ }^{15}$ en una revisión sistemática y meta-análisis de 63 estudios con más de 14.000 individuos en RC, y seguimiento a un año, reportan una reducción significativa de mortalidad $\mathrm{CV}$ en mujeres $\mathrm{y}$ hombres (RR: 0.74; 95\% IC:0.64-0.86). Adicionalmente, se reporta que las mujeres que completan un programa de RC pueden obtener mejores resultados en mortalidad en comparación a los hombres, lo cual no está del todo confirmado ${ }^{16}$. En relación a enfermedad microvascular, Szot y col ${ }^{17}$ objetivaron, que mujeres portadoras de angina microvascular, presentan mejoría clínica y de la perfusión miocárdica en estudio de cintigrafía al término de los programas de RC. A pesar de toda esta evidencia, la $\mathrm{RC}$ es subutilizada en mujeres, siendo éstas menos referidas a los programas de rehabilitación en comparación a los hombres (OR 0.74, 95\% IC:0.69-0.79), y a su vez, aquellas que son referidas y se incorporan a los programas de RC, son menos adherentes y completan en menor porcentaje que los hombres sus sesiones (OR 0.73, 95\% IC:0.68-0.81) ${ }^{18}$.

Se han identificado barreras que limitan la probabilidad de que las mujeres participen en programas de RC, tales como el bajo nivel educacional, la falta de apoyo social, comorbilidades múltiples, y la alta carga de responsabilidades familiares ${ }^{19}$. Por otra parte, son determinantes de mayor participación: un mayor ingreso económico en el hogar, más años de escolaridad (>12 años) y una mejor disposición a asistir a $\mathrm{RC}^{18,19}$.

Existe un llamado de atención sobre que la mayoría de los estudios de revisión sistemática en relación a $\mathrm{RC}$, presentan un desbalance de participación entre hombres y mujeres, por lo que se sugiere que los investigadores incluyan a futuro un número equilibrado de hombres y mujeres, para poder realizar análisis basados en género para objetivar si existe o no disparidad.

En relación a la promoción de actividad física (AF) en mujeres con antecedente de CI, esta dependerá de la estabilidad de su enfermedad de base y su acondicionamiento físico ${ }^{20}$. La recomendación de AF utilizada es la misma para mujeres y hombres, y es la clásica de guía AHA-2011 que indica realizar al menos 150 minutos de intensidad moderada o 75 minutos vigorosa a la semana. La actividad aeróbica debe tener una duración de al menos 10 minutos continuos ${ }^{21}$. (Clase I, Nivel evidencia B). Se debe aconsejar sobre los beneficios $\mathrm{CV}$ adicionales de aumentar la práctica de ejercicio moderado o vigoroso, 300 o 180 minutos a la semana, respectivamente (Clase I, nivel de evidencia B). La práctica de ejercicio aeróbico combinado con fortalecimiento muscular incorporando grandes masas musculares debe realizarse al menos 2 días a la semana. (Clase I, nivel de evidencia B ${ }^{21}$.

\section{Cesación de Tabaco:}

El tabaquismo es un importante factor de riesgo de $\mathrm{CI}$ en la mujer. Una revisión sistemática con meta-análisis de estudios prospectivos de cohortes estratificadas por sexo, demostró que el fumar confiere mayor riesgo coronario en las mujeres en comparación a los hombres. Así, se describe que una mujer fumadora pierde el doble de años versus a un hombre fumador, ocurriendo en ella más prematuramente un infarto al miocardio (13,7 vs 6.2 años; $\mathrm{p}<0,001)^{22}$.

El cese de fumar es una medida de prevención secundaria prioritaria, y tremendamente costo-efectiva tanto en mujeres como en hombres ${ }^{23}$. Critchley y Capewell ${ }^{24}$ han demostrado luego de una revisión sistemática de la literatura, que en población portadora de enfermedad coronaria, el dejar de fumar se asocia a una disminución significativa en el riesgo de mortalidad global (OR:0.64; 95\% IC:0.58-0.71) y de infarto miocárdico (OR:0.68; 95\% IC:0.57-0.82) comparada con el no dejar de fumar, independiente del sexo y la edad del paciente. De este modo, el cese del tabaco tiene una recomendación con nivel de evidencia alto (Clase I, nivel A). En relación al cumplimiento de los programas de cesación y éxito en abolir el hábito tabáquico, la evidencia obtenida con estudios de eficacia y efectividad, como estudios prospectivos y observaciones de recaída, demuestran que las mujeres tienen mayor grado de dificultad de mantener un período largo de abstinencia versus los hombres ${ }^{25}$. Esta información ha abierto una puerta a considerar que las diferencias de género en referencia al cese del tabaco, 
encuentran un criterio de disparidad, y podrían verse influido por factores bio-psico-sociales. Independiente de lo anterior, el clínico debe insistir en el cese del tabaquismo y apoyar si es necesario con terapia coadyuvante para el período de abstinencia.

\section{Prevención Secundaria en Cardiopatía Isquémica: Recomendaciones Farmacológicas}

\section{A. Antiagregantes plaquetarios y Anticoagulantes}

El evitar la formación de trombos intra-arteriales es uno de los pilares de la terapia farmacológica en prevención $\mathrm{CV}$, para lo cual se privilegian los antiagregantes plaquetarios en terapia único o dual.

- Terapia única: Ácido Acetil Salicílico o Antagonistas del Receptor de ADP P2Y12 (Clopidogrel).

Estos constituyen uno de los principales agentes terapéuticos en la prevención de eventos isquémicos recurrentes en pacientes con enfermedad aterosclerótica establecida. Esto se ha demostrado ampliamente en las publicaciones del grupo Antithrombotic Trialists'Collaboration, que incluyen más de 130.000 publicaciones y 50 estudios. Estos estudios han demostrado el efecto cardioprotector de los antiplaquetarios en variadas poblaciones con enfermedad aterosclerótica, especialmente en aquellos con historia de infarto, SCA, accidente vascular encefálico (AVE), y revascularización coronaria y carotidea, y han demostrado beneficios similares en hombres y mujeres $(\text { Clase I nivel A })^{26-29}$.

- Terapia antiplaquetaria doble: Ácido Acetil Salicílico más Antagonistas del Receptor de ADP P2Y12.

Los estudios randomizados relacionados a este tema han enrolado un número menor de mujeres, de este modo, la información referente al análisis de sensibilidad sexo-género específicos sobre eficacia es escasa. Así, en el estudio CURE ("Clopidogrel in Unstable Angina to Prevent Recurrent Ischemic Events") hubo una reducción de riesgo absoluto y relativo en el objetivo compuesto de muerte $\mathrm{CV}$, infarto fatal o no fatal o AVE en el seguimiento a 1 año en mujeres, en comparación a los hombres tratados con clopidogrel más acido acetilsalicílico (AAS), versus AAS sola. En contraste, el CREDO ("Clopidogrel for the Reduction of Events during Observation trial") demostró mayor reducción de eventos en mujeres. Pero el CLARITY (Clopidogrel as Adjunctive Reperfusion Therapy-Thrombolysis in Myocardial Infarction) no demostró diferencias por sexo, incluso con mayor número de even- tos en la rama con clopidogrel + AAS y AAS sola, después de un infarto con supradesnivel ST trombolizado ${ }^{30,31}$. Estos mismos resultados se obtuvieron en el COMMIT ("Clopidogrel and Metoprolol in Myocardial Infarction"). El estudio CHARISMA (Clopidogrel and Aspirin versus Aspirin Alone for the Prevention of Atherothrombotic Events), a diferencia de los anteriores no demostró beneficios en hombres ni mujeres en la combinación clopidogrel + AAS versus AAS solo en sujetos asintomáticos con al menos 1 territorio vascular comprometido y/o 3 o más FR tradicionales. Para aclarar estos resultados conflictivos del beneficio de clopidogrel, un meta-análisis de todos estos estudios demostró que no habían diferencias sexo-género específicas, con similar respuesta en hombres y mujeres 27,32 . Con respecto a qué P2Y12 usar, la evidencia ha demostrado que el ticagrelor + AAS versus clopidogrel + AAS en el estudio PLATO ("PLATelet Inhibition and Patient Outcomes trial") resultó ser superior en cuanto a reducción de eventos. En el análisis de subgrupo sexo-género específico no se demostró diferencia significativa entre $\operatorname{sexos}^{28}$.

Actualmente, en ambos, hombres y mujeres se aconseja la terapia dual: ticagrelor o clopidogrel o plasugrel asociados a AAS en prevención secundaria de SCA, en que se ha realizado una intervención percutánea invasiva con instalación de un stent durante un tiempo mínimo de 1 año. En aquellos en que se ha realizado una intervención percutánea pero en sindrome coronario crónico (SCC) estable, se aconseja una terapia mínima de 6 meses. No existen trabajos que diferencien resultados en hombres y mujeres específicamente. Si cabe considerar que el estudio GRACE de vida real mostró de 2.7 a $4.7 \%$ mayor tasa de sangrado en mujeres después de la instalación de un stent, indicando que el ser mujer era un factor de riesgo para más sangrado $31,33,34$. Finalmente, el estudio PEGASUS (Prevention of Cardiovascular Events in Patients with Prior Heart Attack Using Ticagrelor Compared to Placebo on a Background of AspirinThrombolysis in Myocardial Infarction 54) en pacientes, hombres o mujeres con infarto previo de 1 a 3 años, el tratamiento con ticagrelor más AAS demostró menor riesgo de muerte $\mathrm{CV}$, infarto al miocardio y AVE isquémico, pero también a expensas de mayor sangrado 35 .

\section{- Terapia Triple}

Combinación de anticoagulantes y terapia antiplaquetaria dual después de una intervención coronaria percutánea en pacientes con Fibrilación Auricular u otra indica- 
Figura 1. Prevención secundaria de cardiopatía isquémica en la mujer.

\section{PREVENCION SECUNDARIA EN CARDIOPATIA ISQUEMICA}

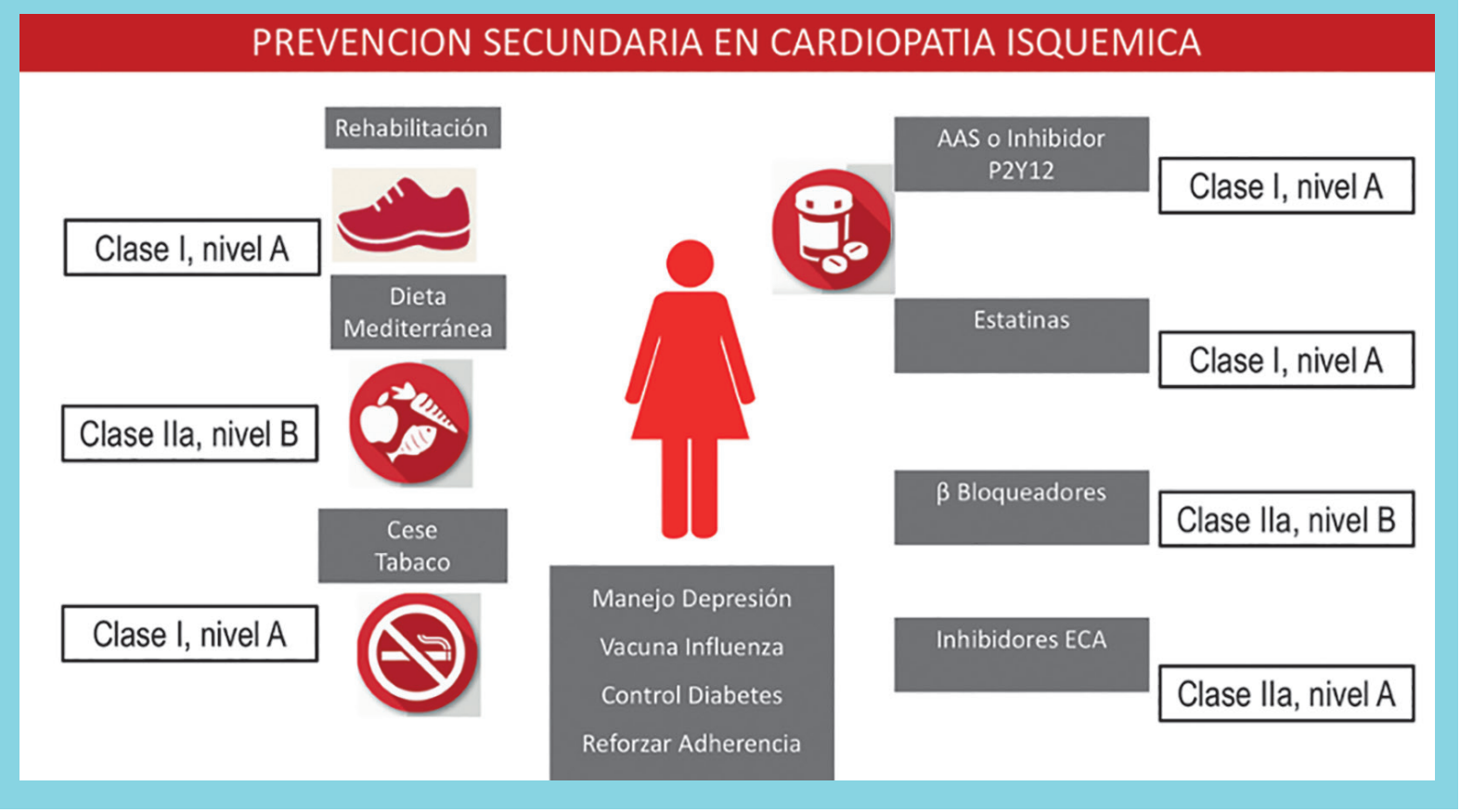

ción de anticoagulación

Se recomienda la terapia anticoagulante antagonista vitamina $\mathrm{K}$ (ej.: warfarina) o anticoagulantes directos (DOAC) en personas con fibrilación auricular post SCA, quienes han recibido un stent, y en pacientes con SCC (en quienes no existe evidencia, pero se actúa igual que post SCA e instalación de stent) para reducir el riesgo de AVE isquémico y eventos $\mathrm{CV}$ futuros. El estudio AUGUSTUS (An Open-label, 2x2 Factorial, Randomized Controlled, Clinical Trial to Evaluate the Safety of Apixaban vs. Vitamin K Antagonist and Aspirin vs. Aspirin Placebo in Patients With Atrial Fibrillation and Acute Coronary Syndrome or Percutaneous Coronary Intervention) ${ }^{36}$ demostró que apixaban $5 \mathrm{mg}$ cada 12 horas se asoció a menor sangrado que la warfarina. Así mismo, AAS versus placebo, mostró mayor sangrado que el placebo. La combinación más segura fue apixaban más placebo, además de un P2Y12, preferentemente, clopidogrel. Sin embargo, hubo más trombosis de stent. La recomendación actual en estos pacientes, hombres y mujeres es que si predomina el riesgo isquémico, se use la triple terapia (anticoagulante oral, AAS y clopidogrel) por $\geq 1$ mes, para evitar la trombosis del stent. No hay evidencia suficiente para el uso de triple terapia aún con ticagrelor y/o plasugrel ${ }^{28}$.

Se ha recomendado que dado la mayor tendencia a complicaciones hemorrágicas de las mujeres, se prefiera un
DOAC versus warfarina ya que son efectivos, y presentan menor riesgo de sangrado en mujeres. Si se usa warfarina, el INR debe ser de 2.0 a 2.5 con un tiempo en rango terapéutico $>70 \% 36,37$.

\section{Dosis baja de Rivaroxabán}

En el Estudio COMPASS (Cardiovascular Outcomes for People Using Anticoagulation Strategies), en pacientes portadores de enfermedad coronaria estable, el uso de rivaroxaban en dosis bajas, de $2.5 \mathrm{mg}$ cada 12 horas, más AAS comparado a AAS sola, demostró menor incidencia de infarto no fatal, accidente vascular encefálico y muerte $\mathrm{CV}$, pero mayor sangrado. El estudio GEMINI-ACS 1(A Study to Compare the Safety of Rivaroxaban Versus Acetylsalicylic Acid in Addition to Either Clopidogrel or Ticagrelor Therapy in Participants With Acute Coronary Syndrome) mostró seguridad con respecto al rivaroxaban en sujetos estables después de un SCA. La reducción del riesgo de eventos en estos dos estudios fue mayor en pacientes de mayor riesgo, como los diabéticos, nefrópatas y con enfermedad vascular periférica. No se hace mención a diferencias sexo-género específicas 38,39 .

\section{B. Control de Lípidos}

El control de los lípidos es relevante en la prevención de la CI, focalizado principalmente en la reducción del colesterol LDL. Dentro de las alternativas terapéuticas 
para reducir éste, la familia de las estatinas ha sido la más eficiente en lograr este objetivo. Estudios randomizados con estatinas han objetivado que la reducción del col LDL se asocia directamente con la reducción de eventos coronarios y mortalidad $\mathrm{CV}$ en sujetos portadores de enfermedad coronaria aterosclerótica, independiente de su género. Es así como en población con enfermedad vascular conocida, una reducción del col LDL en $39 \mathrm{mg} /$ dl (= $1 \mathrm{mmol} / \mathrm{L})$ bajo la acción de una estatina, reduce significativamente la aparición de eventos $\mathrm{CV}$ mayores tanto en mujeres (RR 0,84, 99\% CI:0,77-0,91) como en hombres (RR 0,79, 99\% CI:0,76- 0,81) ${ }^{40}$.

Basado en lo anterior, las estatinas se recomiendan para prevención secundaria en mujeres con las mismas indicaciones y metas terapeúticas que en los hombres, con un nivel alto de evidencia (Clase I, nivel A) ${ }^{41}$. Se recomiendan de preferencia dos estatinas por su potencia en dosis alta: atorvastatina (40 o $80 \mathrm{mg}$ ) y rosuvastatina (20 o 40 $\mathrm{mg}$ ) al día. El objetivo terapéutico en la reducción del col LDL es lograr un valor de menos de $<70 \mathrm{mg} / \mathrm{dL}$ y/o una reducción de un $\geq 50 \%$ del valor basal. La reciente recomendación de la Sociedad Europea de Cardiología es más exigente, aspirando a un descenso a $<55 \mathrm{mg} / \mathrm{dL}$, y en pacientes con enfermedad ateroesclerótica con historia de más de un evento vascular en los dos años siguientes del primero, podría ser considerado un objetivo $<40 \mathrm{mg} / \mathrm{dL}$, muy difícil de lograr sin el apoyo de terapia farmacológica asociada a la estatina ${ }^{42}$.

La indicación de ezetimibe en pacientes que no logran alcanzar el objetivo terapéutico con estatina, está fundamentada en un sólo estudio randomizado que demostró beneficio al adicionar ezetimibe a la simvastatina posterior a un SCA (Clase I, nivel B). Se objetivó reducción de riesgo en el objetivo primario combinado, no en mortalidad. En un subestudio con análisis de resultados sexoespecíficos, se obtuvo un $12 \%$ de reducción de riesgo en el objetivo primario en mujeres. Si sólo consideramos el número total del primer evento post SCA, las mujeres presentaron una reducción significativa de un $18 \%$ con la adición de ezetimibe (RR 0.81:IC 95\%: 0.71-0.94) y los hombres una reducción de un 6\% (RR 0.94:IC 95\%: 0.87-1.02) 43 .

En relación a los anti PCSK9 para prevención secundaria isquémica en la mujer, es necesario considerar que sólo $25 \%$ de los participantes han sido mujeres en los estudios de eventos CV con alirocumab y evelocumab 41,42 . En ambos estudios hubo eficacia en aquellos sujetos que recibieron el fármaco activo, observándose una reducción significativa de eventos cardiovasculares en ambos sexos. De este modo, esta terapia está recomendada en hombres y mujeres que no logran su objetivo terapéutico a pesar de estar recibiendo estatina en alta dosis (Clase I, nivel A) ${ }^{41,42}$. No existen publicaciones con análisis sexo-específico con estos fármacos. En Chile todavía no se dispone de esta terapia, y su prescripción dependerá del acceso a ésta.

\section{B-bloqueadores ( $\boldsymbol{\beta B}$ )}

Los $\beta \mathrm{B}$ se han considerado un pilar fundamental en la prevención secundaria para los pacientes postinfarto, basado en los estudios randomizados en las décadas de 1970 y 1980, que demostraron un gran beneficio en la mortalidad. Es así, como un metaanálisis que estudió el uso prolongado de $\beta \mathrm{B}$ en el riesgo de muerte, demostró un $23 \%$ de reducción de mortalidad, fundamentalmente, de muerte súbita $(32 \%)^{28,44}$.

El uso de $\beta \mathrm{B}$ post hospitalización por infarto al miocardio se ha convertido en una medida de calidad de cuidado en salud, y hoy se prescribe $90 \%$ en USA como en Europa. Los $\beta \mathrm{B}$ son beneficiosos, sin duda en la prevención de muerte por insuficiencia cardíaca y disfunción ventricular izquierda. Sin embargo, en el tratamiento del SCC posee menos evidencia ${ }^{28}$. Hoy sus indicaciones son 2 :

a) en el tratamiento crónico para aliviar la angina.

b) para reducir eventos cardiovasculares en pacientes con SCC.

Esta última indicación, sin embargo, no se basa en la evidencia en prevención secundaria.

La principal evidencia en SCC proviene de Bangalore y cols., usando la población del registro REACH. Ellos evaluaron dos clases de pacientes con SCC: un grupo con infarto < de 1 año al enrolamiento, y el otro grupo sin infarto previo. Haciendo "propensity score matching" demostraron que el uso de $\beta \mathrm{B}$ no redujo el infarto al miocardio ni cerebral, tanto fatal y no fatal, ni la muerte cardíaca. En otro estudio, usando el registro CORONOR, se demostró que los $\beta \mathrm{B}$ reducían el riesgo de muerte, pero con una población preferentemente de hombres 45 .

Lamentablemente, toda la evidencia en $\beta \mathrm{B}$ viene de cohortes mixtas, por lo cual no hay evidencia género-específica.

Teniendo en consideración lo que se ha discutido, las indicaciones del uso actual de $\beta \mathrm{B}$, y su nivel de evidencia son: 1. Alivio de la angina en pacientes con SCC. (IA)

2. Pacientes que han tenido un infarto y cursan con disfunción ventricular izquierda o falla cardíaca establecida 
post infarto, para disminuir morbilidad y mortalidad. (IA) 3. A largo plazo en pacientes post infarto podrían usarse. (IIaB) Los $\beta \mathrm{B}$ deben ser titulados según la frecuencia cardíaca del paciente para llegar a 50-60 latidos por minutos. Para el alivio de la angina se pueden usar todos los $\beta \mathrm{B}$.

\section{Inhibidores de la Enzima Convertidora y Bloquea- dores de Receptores de Angiotensina 2}

Los inhibidores de la enzima convertidora (IECA) tienen plena recomendación de uso en pacientes portadores de insuficiencia cardíaca con disfunción sistólica del ventrículo izquierdo, basado en estudios que han demostrado reducción de morbi-mortalidad cardiovascular (Clase I, nivel A). Del mismo modo, tienen fuerte indicación post SCA en aquel paciente de alto riesgo, caracterizado por tener infarto de pared anterior, historia previa de infarto, o insuficiencia cardíaca clínica (Clase I, nivel A) ${ }^{28}$.

El uso de IECA como medida de prevención secundaria en sujetos con enfermedad coronaria estable sin insuficiencia cardíaca ha demostrado información más discordante. En las revisiones sistemáticas siempre se analizan seis estudios con enfermedad coronaria estable, que incluye al estudio Heart Outcomes Prevention Evaluation (HOPE) que también incorporó diabéticos. Meta-análisis de estos estudios, entre los cuales un 15 a $28 \%$ de la población correspondían a mujeres, han demostrado que el uso de IECA se asocia a una disminución de mortalidad CV (RR:0.83; IC 95\%: 0.72-0.96), e infarto no fatal \% (RR:0.84; IC 95\%: 0.75-0.94) 46,47 . No obstante no todos los estudios por separado han objetivado reducción significativa de eventos CV o mortalidad. De este modo la indicación de IECA en prevención secundaria en CI tiene una recomendación IIa- $\mathrm{A}^{28}$. No hay publicaciones con análisis sexo-específico con IECA en prevenciónn secundaria coronaria. Sólo recordar que estos fármacos son potencialmente teratogénicos y no se indican en mujeres fértiles sin protección anticonceptiva.

El uso de bloqueadores de receptor de angiotensina II tiene menor evidencia, siendo válido como una alternativa ante la intolerancia a los IECA ${ }^{47}$. Bangalore y $\operatorname{col}^{48}$, en su reciente meta-análisis en pacientes con SCC, concluyen que los inhibidores del eje renina-angiotensina, tanto IECA como bloqueadores de receptor de angiotensina II, reducen eventos $\mathrm{CV}$ y muerte cuando el comparador es placebo, no así cuando el comparador es una control activo. A la luz de esta información no es recomendable su indicación en la ausencia de insuficiencia cardíaca o de un alto riesgo CV (ej.: diabetes).

\section{E. Control de la Diabetes}

Las anormalidades de la glicemia son comunes en los pacientes con SCA y cardiopatía isquémica estable, y se asocian a un peor pronóstico CV. Entre un 20-30\% de los pacientes con cardiopatía isquémica tienen diabetes, y el $70 \%$ restante presenta alteraciones en el test de tolerancia, ya sea nueva diabetes o intolerancia a la glucosa. La mujer diabética con CI tiene peor pronóstico que el hombre, reportándose un $50 \%$ más de probabilidad de cursar con eventos coronarios fatales. Por lo anterior, el estado glicémico en las mujeres con CI debe investigarse, y si son diabéticas, se debe cumplir con una estricta prevención secundaria.

Con respecto al tratamiento, durante un SCA se ha demostrado que el control glicémico intensivo con insulina reduce la mortalidad. Desgraciadamente no existen estudios randomizados que demuestren en pacientes coronarios, los eventuales beneficios de la metformina o sulfonilureas. La mayoría son estudios en subpoblaciones de diabéticos, sin enfermedad aterosclerótica previa, como el UKPDS ${ }^{49}$.

En los últimos años, y por primera vez en la historia de la diabetes, se dan a conocer varios estudios randomizados con puntos finales duros y con nuevos hipoglicemiantes en pacientes con enfermedad $\mathrm{CV}$ o con alto/muy alto riesgo $\mathrm{CV}$. Los resultados de los estudios con análogos de GLP-1 (estudios LEADER, SUSTAIN-6, Harmony Outcomes, REWIND, y PIONEER 6) y con los inhibidores de SGLT2 (EMPA-REG OUTCOME, CANVAS, DECLARE-TIMI 58, y CREDENCE) demostraron que estas drogas debieran ser recomendadas en pacientes diabéticos con enfermedad cardiovascular prevalente o sujetos de alto/muy alto riesgo $\mathrm{CV}$, como los con daño de órgano blanco o múltiples factores de riesgo $\mathrm{CV}$, ya sea que estén o no con metformina. Análisis genero específico de los inhibidores de SGLT2 demuestran igual protección $\mathrm{CV}^{49}$.

Conforme a las últimas guías europeas de diabetes el control glicémico debiera seguirse con $\mathrm{HbA} 1 \mathrm{C}<7 \%$ para la mayoría de los adultos; $<6.5 \%$ si se puede llegar a ese objetivo sin hipoglicemias $\mathrm{y}<8 \%$ en adultos mayores 49 .

\section{F. Factores Psicosociales}

Adicionalmente, la mujer con EC en comparación al hombre tiene mayor prevalencia de trastornos asociadas al ánimo como son el estrés y la depresión, que inciden negativamente en la CI. Varios mecanismos biológicos asocian a la depresión con un peor pronóstico de la EC tales como son: una mayor activación inflamatoria, mayor reactividad plaquetaria, un desbalance autonómico, y alteración en la 
arquitectura del sueño, pero sin duda la situación más perturbadora es la alteración conductual de un desinterés en mantener un estilo de vida saludable junto con una mala adherencia al tratamiento farmacológico. Es así como entre pacientes post SCA, Rieckman y col reportan que aproximadamente un $42 \%$ de pacientes depresivos toman AAS menos de un $75 \%$ del tiempo, vs un $10 \%$ de no depresivos. De este modo, es fundamental una adecuada pesquisa del trastorno del ánimo y precoz tratamiento ${ }^{50}$.

Finalmente, no sólo es fundamental la adecuada prescripción de fármacos, sino también la adherencia por parte de la paciente. Lamentablemente, menos del 50\% de los pacientes son totalmente adherentes a todos los medicamentos con indicación clase I en prevención secundaria, lo que se asocia a un aumento en el riesgo de eventos $\mathrm{CV}$ recurrentes y muerte ${ }^{28}$.

\section{G. Otros}

La prevención secundaria de CI en la mujer debe incorporar otras medidas las cuales no debemos olvidar, como la vacunación anual para virus de la influenza, que es una indicación de tipo $\mathrm{IB}^{28}$.

Por todo lo anteriormente discutido, la formación de programas de prevención secundaria ambulatorios, equipos multidisciplinarios formados por cardiólogos, enfermeros, nutricionistas, kinesiólogos, y psicólogos son deseables para tratar estos pacientes con enfermedad ateroesclerótica isquémica. Estos programas tienen como objetivo fundamental tratar en forma agresiva todos los factores de riesgo $\mathrm{CV}$, manteniendo la adherencia de los pacientes no sólo a sus medicamentos, sino a estilos de vida saludable y programas de rehabilitación cardiovascular.

\section{References:}

1. DEPARTAMENTO DE SALUD Y ESTADÍSTICAS VITALES 2016; htps://webanterior.ine.cl/estadisticas/demograficas-y-vitales. Acceso Julio 2020.

2. DICKERSON JA, NAGARAJA HN, RAMAN SV. Gender-related differences in coronary artery dimensions: a volumetric analysis. Clin Cardiol. 2010;33(2):E44-9.

3. SHARAF B, WOOD T, SHAW L, JOHNSON BD, KELSEY $\mathrm{S}$, ANDERSON RD, et al. Adverse outcomes among women presenting with signs and symptoms of ischemia and no obstructive coronary artery disease: findings from the National Heart, Lung, and Blood Institute-sponsored Women's Ischemia Syndrome Evaluation (WISE) angiographic core laboratory. Am Heart J. 2013;166(1):134-41.

4. MEHTA LS, BECKIE TM, DEVON HA, GRINES CL, KRUMHOLZ HM, JOHNSON MN, et al. Acute Myocardial Infarction in Women: A Scientific Statement From the American Heart Association. Circulation. 2016;133(9):916-47.

5. UDELL JA, KOH M, QIU F, AUSTIN PC, WIJEYSUNDERA HC, BAGAI A, et al. Outcomes of Women and Men With Acute Coronary Syndrome Treated With and Without Percutaneous Coronary Revascularization. J Am Heart Assoc. 2017;6(1).
6. ALABAS OA, GALE CP, HALL M, RUTHERFORD MJ, SZUMMER K, LAWESSON SS, et al. Sex Differences in Treatments, Relative Survival, and Excess Mortality Following Acute Myocardial Infarction: National Cohort Study Using the SWEDEHEART Registry. J Am Heart Assoc. 2017;6(12).

7. LEE KK, FERRY AV, ANAND A, STRACHAN FE, CHAPMAN AR, KIMENAI DM, et al. Sex-Specific Thresholds of High-Sensitivity Troponin in Patients With Suspected Acute Coronary Syndrome. J Am Coll Cardiol. 2019;74(16):2032-43.

8. DE LORGERIL M, SALEN P, MARTIN JL, MAMELLE $\mathrm{N}$, MONJAUD I, TOUBOUL $\mathrm{P}$, et al. Effect of a mediterranean type of diet on the rate of cardiovascular complications in patients with coronary artery disease. Insights into the cardioprotective effect of certain nutriments. J Am Coll Cardiol. 1996;28(5):1103-8.

9. PIEPOLI MF, HOES AW, AGEWALL S, ALBUS C, BROTONS C, CATAPANO AL, et al. 2016 European Guidelines on cardiovascular disease prevention in clinical practice: The Sixth Joint Task Force of the European Society of Cardiology and Other Societies on Cardiovascular Disease Prevention in Clinical Practice (constituted by representatives of 10 societies 
and by invited experts)Developed with the special contribution of the European Association for Cardiovascular Prevention \& Rehabilitation (EACPR). Eur Heart J. 2016;37(29):2315-81.

10. ESTRUCH R. Anti-inflammatory effects of the Mediterranean diet: the experience of the PREDIMED study. Proc Nutr Soc. 2010;69(3):333-40.

11. URPI-SARDA M, CASAS R, CHIVA-BLANCH G, ROMERO-MAMANI ES, VALDERAS-MARTINEZ P, ARRANZ S, et al. Virgin olive oil and nuts as key foods of the Mediterranean diet effects on inflammatory biomakers related to atherosclerosis. Pharmacol Res. 2012;65(6):577-83.

12. REES K, TAKEDA A, MARTIN N, ELLIS L, WIJESEKARA D, VEPA A, et al. Mediterranean-style diet for the primary and secondary prevention of cardiovascular disease. Cochrane Database Syst Rev. 2019;3:CD009825.

13. ANDERSON JW, KENDALL CW, JENKINS DJ. Importance of weight management in type 2 diabetes: review with meta-analysis of clinical studies. J Am Coll Nutr. 2003;22(5):331-9.

14. STEVENS VJ, CORRIGAN SA, OBARZANEK E, BERNAUER E, COOK NR, HEBERT P, et al. Weight loss intervention in phase 1 of the Trials of Hypertension Prevention. The TOHP Collaborative Research Group. Arch Intern Med. 1993;153(7):849-58.

15. ANDERSON L, OLDRIDGE N, THOMPSON DR, ZWISLER AD, REES K, MARTIN N, et al. Exercise-Based Cardiac Rehabilitation for Coronary Heart Disease: Cochrane Systematic Review and Meta-Analysis. J Am Coll Cardiol. 2016;67(1):1-12.

16. SANDERSON BK, SHEWCHUK RM, BITTNER V. Cardiac rehabilitation and women: what keeps them away? J Cardiopulm Rehabil Prev. 2010;30(1):12-21.

17. SZOT W, ZAJAC J, KUBINYI A, KOSTKIEWICZ M. The effects of cardiac rehabilitation on overall physical capacity and myocardial perfusion in women with microvascular angina. Kardiol Pol. 2016;74(5):431-8.

18. SUPERVIA M, MEDINA-INOJOSA JR, YEUNG C, LOPEZ-JIMENEZ F, SQUIRES RW, PEREZ-TERZIC CM, et al. Cardiac Rehabilitation for Women: A Systematic Review of Barriers and Solutions. Mayo Clin Proc. 2017.

19. CUGUSI L, MERCURO G. A systematic overview to quantify the gender imbalance in cardiovascular rehabilitation trials. Eur J Prev Cardiol. 2019;26(7):776-81.

20. SATTELMAIR J, PERTMAN J, DING EL, KOHL HW, 3RD, HASKELL W, LEE IM. Dose response between physical activity and risk of coronary heart disease: a meta-analysis. Circu- lation. 2011;124(7):789-95.

21. MOSCA L, BENJAMIN EJ, BERRA K, BEZANSON JL, DOLOR RJ, LLOYD-JONES DM, et al. Effectiveness-based guidelines for the prevention of cardiovascular disease in women--2011 update: a guideline from the american heart association. Circulation. 2011;123(11):1243-62.

22. GRUNDTVIG M, HAGEN TP, GERMAN M, REIKVAM A. Sex-based differences in premature first myocardial infarction caused by smoking: twice as many years lost by women as by men. Eur J Cardiovasc Prev Rehabil. 2009;16(2):174-9.

23. HUXLEY RR, WOODWARD M. Cigarette smoking as a risk factor for coronary heart disease in women compared with men: a systematic review and meta-analysis of prospective cohort studies. Lancet. 2011;378(9799):1297-305.

24. CRITCHLEY J, CAPEWELL S. Smoking cessation for the secondary prevention of coronary heart disease. Cochrane Database Syst Rev. 2004(1):CD003041.

25. SMITH PH, BESSETTE AJ, WEINBERGER AH, SHEFFER CE, MCKEE SA. Sex/gender differences in smoking cessation: A review. Prev Med. 2016;92:135-40.

26. KUMBHANI DJ, STEG PG, CANNON CP, EAGLE KA, SMITH SC, JR., HOFFMAN E, et al. Adherence to secondary prevention medications and four-year outcomes in outpatients with atherosclerosis. Am J Med. 2013;126(8):693-700 e1.

27. ANTITHROMBOTIC TRIALISTS C. Collaborative meta-analysis of randomised trials of antiplatelet therapy for prevention of death, myocardial infarction, and stroke in high risk patients. BMJ. 2002;324(7329):71-86.

28. KNUUTI J, WIJNS W, SARASTE A, CAPODANNO D, BARBATO E, FUNCK-BRENTANO C, et al. 2019 ESC Guidelines for the diagnosis and management of chronic coronary syndromes. Eur Heart J. 2020;41(3):407-77.

29. BRAUNSTEIN JB, KERSHNER DW, BRAY P, GERSTENBLITH G, SCHULMAN SP, POST WS, et al. Interaction of hemostatic genetics with hormone therapy: new insights to explain arterial thrombosis in postmenopausal women. Chest. 2002;121(3):906-20.

30. KUMBHANI DJ, STEG PG, CANNON CP, EAGLE KA, SMITH SC, JR., HOFFMAN E, et al. Adherence to secondary prevention medications and four-year outcomes in outpatients with atherosclerosis. Am J Med. 2013;126(8):693-700.e1.

31. BASILI S, RAPARELLI V, PROIETTI M, TANZILLI G, FRANCONI F. Impact of sex and gender on the efficacy of antiplatelet therapy: the female perspective. J Atheroscler 
Thromb. 2015;22(2):109-25.

32. BERGER JS, BHATT DL, CANNON CP, CHEN Z, JIANG L, JONES JB, et al. The relative efficacy and safety of clopidogrel in women and men a sex-specific collaborative meta-analysis. J Am Coll Cardiol. 2009;54(21):1935-45.

33. FRANCONI F, CARRU C, MALORNI W, VELLA S, Mercuro G. The effect of sex/gender on cardiovascular pharmacology. Curr Pharm Des. 2011;17(11):1095-107.

34. WANG TY, ANGIOLILLO DJ, CUSHMAN M, SABATINE MS, BRAY PF, SMYTH SS, et al. Platelet biology and response to antiplatelet therapy in women: implications for the development and use of antiplatelet pharmacotherapies for cardiovascular disease. J Am Coll Cardiol. 2012;59(10):891-900.

35. BONACA MP, BHATT DL, COHEN M, STEG PG, STOREY RF, JENSEN EC, et al. Long-term use of ticagrelor in patients with prior myocardial infarction. $\mathrm{N}$ Engl $\mathrm{J}$ Med. 2015;372(19):1791-800.

36. LOPES RD, HEIZER G, ARONSON R, VORA AN, MASSARO T, MEHRAN R, et al. Antithrombotic Therapy after Acute Coronary Syndrome or PCI in Atrial Fibrillation. N Engl J Med. 2019;380(16):1509-24.

37. PANCHOLY SB, SHARMA PS, PANCHOLY DS, PATEL TM, CALLANS DJ, MARCHLINSKI FE. Meta-analysis of gender differences in residual stroke risk and major bleeding in patients with nonvalvular atrial fibrillation treated with oral anticoagulants. Am J Cardiol. 2014;113(3):485-90.

38. CONNOLLY SJ, EIKELBOOM JW, BOSCH J, DAGENAIS G, DYAL L, LANAS F, et al. Rivaroxaban with or without aspirin in patients with stable coronary artery disease: an international, randomised, double-blind, placebo-controlled trial. Lancet. 2018;391(10117):205-18.

39. OHMAN EM, ROE MT, STEG PG, JAMES SK, POVSIC TJ, WHITE J, et al. Clinically significant bleeding with low-dose rivaroxaban versus aspirin, in addition to P2Y12 inhibition, in acute coronary syndromes (GEMINI-ACS-1): a double-blind, multicentre, randomised trial. Lancet. 2017;389(10081):1799-808.

40. CHOLESTEROL TREATMENT TRIALISTS C, FULCHER J, O'CONNELL R, VOYSEY M, EMBERSON J, BLACKWELL L, et al. Efficacy and safety of LDL-lowering therapy among men and women: meta-analysis of individual data from 174,000 participants in 27 randomised trials. Lancet. 2015;385(9976):1397-405.

41. GRUNDY SM, STONE NJ, BAILEY AL, BEAM C, BIRTCHER KK, BLUMENTHAL RS, et al. 2018 AHA/ACC/AACVPR/AAPA/ABC/ACPM/ADA/AGS/APhA/ASPC/NLA/ PCNA Guideline on the Management of Blood Cholesterol: A
Report of the American College of Cardiology/American Heart Association Task Force on Clinical Practice Guidelines. Circulation. 2019;139(25):e1082-e143.

42. MACH F, BAIGENT C, CATAPANO AL, KOSKINAS KC, CASULA M, BADIMON L, et al. 2019 ESC/EAS Guidelines for the management of dyslipidaemias: lipid modification to reduce cardiovascular risk. Eur Heart J. 2020;41(1):111-88.

43. KATO ET, CANNON CP, BLAZING MA, BOHULA E, GUNERI S, WHITE JA, et al. Efficacy and Safety of Adding Ezetimibe to Statin Therapy Among Women and Men: Insight From IMPROVE-IT (Improved Reduction of Outcomes: Vytorin Efficacy International Trial). J Am Heart Assoc. 2017;6(11).

44. BANGALORE S, STEG G, DEEDWANIA P, CROWLEY K, EAGLE KA, GOTO S, et al. beta-Blocker use and clinical outcomes in stable outpatients with and without coronary artery disease. JAMA. 2012;308(13):1340-9.

45. BAUTERS C, LEMESLE G, MEURICE T, TRICOT O, DE GROOTE P, LAMBLIN N. Prognostic impact of $\beta$-blocker use in patients with stable coronary artery disease. Heart. 2014;100(22):1757-61.

46. EFFECTS OF RAMIPRIL ON CARDIOVASCULAR AND MICROVASCULAR OUTCOMES IN PEOPLE WITH DIABETES MELLITUS: results of the HOPE study and MICRO-HOPE substudy. Heart Outcomes Prevention Evaluation Study Investigators. Lancet. 2000;355(9200):253-9.

47. AL-MALLAH MH, TLEYJEH IM, ABDEL-LATIF AA, WEAVER WD. Angiotensin-converting enzyme inhibitors in coronary artery disease and preserved left ventricular systolic function: a systematic review and meta-analysis of randomized controlled trials. J Am Coll Cardiol. 2006;47(8):1576-83.

48. BANGALORE S, FAKHERI R, WANDEL S, TOKLU B, WANDEL J, MESSERLI FH. Renin angiotensin system inhibitors for patients with stable coronary artery disease without heart failure: systematic review and meta-analysis of randomized trials. BMJ. 2017;356:j4.

49. GRANT PJ, COSENTINO F. The 2019 ESC Guidelines on diabetes, pre-diabetes, and cardiovascular diseases developed in collaboration with the EASD: New features and the 'Ten Commandments' of the 2019 Guidelines are discussed by Professor Peter J. Grant and Professor Francesco Cosentino, the Task Force chairmen. Eur Heart J. 2019;40(39):3215-7.

50. PAJAK A, JANKOWSKI P, KOTSEVA K, HEIDRICH J, DE SMEDT D, DE BACQUER D, et al. Depression, anxiety, and risk factor control in patients after hospitalization for coronary heart disease: the EUROASPIRE III Study. Eur J Prev Cardiol. 2013;20(2):331-40. 